\title{
Study on Wuliangye microblog marketing strategy based on 5W model
}

\author{
Kui Yu
}

College of Economic and Management, Yibin University, Yibin, 64400, China

Keywords: microblog marketing, Wuliangye, liquor

\begin{abstract}
As microblog is widely applied, large quantities of liquor enterprises also adopt this platform for marketing, in the hope of helping liquor enterprises walk out of the plight. This paper discusses Wuliangye microblog marketing strategy under such background, analyzes the necessity and current situation of microblog marketing and analyzes the strategy and method of Wuliangye microblog marketing with the help of " $5 \mathrm{~W}$ " model. It is hoped that the analysis can provide reference for microblog marketing of other liquor enterprises.
\end{abstract}

\section{Introduction}

After 10 golden years, sales status of liquor industry takes a sudden turn and becomes worse rapidly under the background of consumption trend change and severe control of consumption with public funds by the state. Thus, the sale of Wuliangye Group is caught into trouble. According to the data of the first quarter in 2014 disclosed by Wuliangye at Shenzhen Stock Exchange, the business revenue in the reporting period was RMB 6.72 billion, down $22.54 \%$ year on year. The net margin was RMB 2.619 billion, down 27.79\% year on year. It can be seen from the data in the first quarter that performance pressure of Wuliangye is still no improved. This year is a year of adjustment and seeking reform for Wuliangye. Currently, internet marketing (especially microblog marketing) is a good strategy selection for Wuliangye to go out of the plight.

\section{Overview of microblog marketing}

Weibo (MicroBlog for short) is a broadcast-type social networking platform through paying attention to mechanism and sharing short real-time information. The earliest microblog is American twitter. Microblog marketing refers to a marketing mode where enterprises find and meet users' demands via microblog platform and create value for enterprises and individuals.

Twitter - originator of microblog specially sets up "brand channel", constructs brand publicity page for enterprises and organizes various teams to send all kinds of promotion and activity information to users so as to achieve brand promotion through exchange and interaction with users. Dell, Starbucks, KFC, Coca Cola, Ford Motor and other international famous brands carry out microblog marketing ${ }^{[1]}$. UFIDA Software Group, VANCL, Chang'an Ford, China Mobile, China Telecom and Seven Clothing also carry out microblog marketing activities one after another to gather popularity, improve brand influence and become new battle field for marketing through microblog.

\section{Introduction to $5 \mathrm{~W}$ model}

In 1948, American scholar H·Lasswell used "5W model" to analyze information communication process in Structure and Function of Communication in Society. People call this model Lasswell Formula. The following figure displays schematic diagram of $5 \mathrm{~W}$ model.

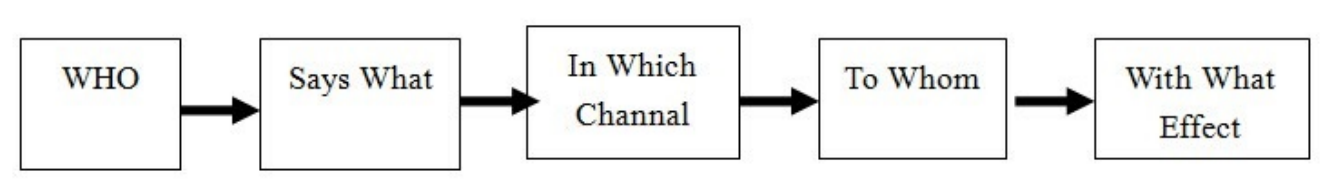


5W: the first $\mathrm{W}$ is "Who" representing communication subject of microblog marketing information; the second $\mathrm{W}$ is "Says What" reflecting information contents communicated by microblog marketing subjects; the third W is "In Which Channel" which means the media used to transmit marketing information; the fourth $\mathrm{W}$ is "To Whom" which means the audiences of microblog marketing information; the fifth $\mathrm{W}$ is "With What Effect" which means effects of microblog marketing. The $5 \mathrm{~W}$ fully reflects the whole process of microblog marketing information dissemination. So, when enterprises carry out microblog marketing, $5 \mathrm{~W}$ model can be used to well manage and control microblog marketing process ${ }^{[2]}$.

\section{Necessity of Wuliangye microblog marketing}

\section{Trend requirement of liquor industry development}

At present, many liquor-making enterprises or individuals set up microblog, including manufacturing enterprises, circulation enterprises, enterprise official microblog and employee microblog. Besides, there is microblog of liquor lovers. Thus, the coverage is very wide. Through simple retrieval in Sina Microblog, we can find Luzhou Laojiao, Gujinggong Liquor and Jing Liquor set up official microblog and boost exposure rate of enterprises in microblog platform. Sina Microblog has a total of 106 microblogs with the title of Luzhou Laojiao, including Luzhou Laojiao head office and local branches. It thus can be seen that microblog marketing has become the trend of liquor industry marketing. Wuliangye marketing development also should follow such trend.

\section{Need for marketing mode change}

Relative to food and beverage marketing, overall marketing level of liquor industry is relatively traditional and lags behind. The marketing means and communication media are single. They mainly depend on television, newspaper and other traditional media platforms for marketing promotion. The generations after 80 s and 90 s become the main force of consumption. Their internet features will inevitably influence enterprise marketing mode with network feature.

According to statistics, in the first half year of 2013, registered users of Sina Microblog reached 536 million. If such huge microblog users transform to product sales market, unimaginable economic benefits will certainly be brought to enterprises. Microblog user group is a high-end group using Chinese internet. Although such part of user group only occupies $10 \%$ of Chinese internet user group, it is the group with the highest purchasing power on Chinese internet. Such group is potential customer group of Wuliangye. So, Wuliangye must attach importance to microblog marketing and open up young high-end group market.

\section{Need for Wuliangye to get rid of plight}

Currently, after consumption of public funds on three major private purposes--cars, banquets and oversea visits is limited, Wuliangye is faced with productivity and inventory. In accordance with the performance of Wuliangye in the first quarter in 2014 published in April 28, the company achieved net margin RMB 2.619 billion in the first quarter, down $27.79 \%$ year on year. Continuous profit growth momentum for many years is restrained. Wuliangye must seek new platform and mew mode to get rid of current plight. As microblog marketing is applied more and more widely, Wuliangye can not just improve brand exposure rate, realize communication reform, but also bind consumers and boost product sales via microblog marketing.

\section{Analysis of Wuliangye microblog marketing}

\section{Current situation of Wuliangye microblog marketing}

According to the data of Sina Microblog on April 29, 2014, there are a total of 3322 microblogs related to Wuliangye set up in Sina Microblog, including 274 authenticated users. There are also individual authenticated users, such as Liu Yu - brand manager of Sales Department in Yibin Wuliangye Co., Ltd. There are also brand authenticated users, such as Wuliangye Bingpo 43, nationwide general dealer of Wuliangye caller liquor, Wuliangye Beijing flagship store Beiyuan branch and Bazhou Xinniang Wuliangye Company. But Wuliangye Co., Ltd. does not set up official microblog. Although Tencent has microblog identification of "Wuliangye Group", it is not official 
but individual.

\section{Influence of Wuliangye microblog marketing}

Individual microblog with large influence is the microblog of "Wuliangye investment attraction - Liu Yu”. He owns 1655 followers, 25376 fans and 11355 articles. The authenticated microblog with large influence is the microblog of "Wuliangye Bingpo 43". He owns 366 followers, 2848 fans and 1158 articles. They still have large gap with 758 followers, 436362 fans and 6291 articles of "Luzhou Laojiao China Glory”, 329 followers, 1429341 fans and 2224 articles of "China Jing Liquor”. In accordance with ranking list of Sina Microblog, in food industry, liquor enterprise with the highest ranking is official microblog of Carlsberg Beer. Among liquor enterprises, China Jing Liquor has the highest ranking. Wuliangye is excluded in the list.

It can be seen from the above analysis that Wuliangye quite lags behind in the aspect of microblog marketing. Thus, it is required to enhance microblog marketing construction.

\section{Analysis of Wuliangye microblog marketing strategy}

" $5 \mathrm{~W}$ " is a significant analysis tool in microblog marketing. " $5 \mathrm{~W}$ " model is used to analyze Wuliangye microblog marketing strategy.

\section{(I) Specify microblog orientation and establish microblog matrix}

"WHO" in the model refers to microblog information publishers publishing microblog topics and enhancing microblog influence. When enterprises carry out microblog marketing, microblog information publishers include two types: 1) enterprise microblog; 2) individual microblog assisting enterprise microblog. The two types of microblog play different roles in microblog marketing and mutually cooperate.

\section{Specify microblog orientation}

According to the orientation, enterprise microblog can be divided into official microblog, product microblog, brand microblog, employee microblog, leadership microblog, recruitment microblog and public relation microblog. Different microblogs contain different contents and styles. Official microblog mainly announces enterprise business information, business performance and news dynamics. Leadership microblog mainly plays the role in guiding public opinions and play the role of industrial guider. Generally speaking, a microblog should not play all roles, which goes against microblog communication and influence. Luzhou Laojiao sets up official microblogs including "Luzhou Laojiao China Glory”, "Luzhou Laojiao Six-year official microblog”. There are also individual microblogs: individual microblog of Yu Rang - director of president office in Luzhou Laojiao Group. There are also sales microblogs: microblog of "Luzhou Laojiao Tonghe Flagship Store”. Different microblogs cooperate mutually.

Wuliangye also should set up various types of microblogs according to different orientations, such as Wuliangye official microblog, Wuliangye Chunhe Wuliangchun brand microblog and Wuliangye distribution Wuliangye. National treasure-class bartenders of Wuliangye can also set up individual microblog and guide industrial public opinions. Meanwhile, Wuliangye can set up microblog with the help of existing liquor museum to introduce the long-standing liquor culture of China.

\section{Establish microblog matrix}

Enterprises should establish multiple microblog identifications and utilize fission-type propagation characteristics of microblog. The communication effects will grow in the form of geometrical progression, like "virus-type" communication. Different cooperation relations of multiple microblog identifications form different microblog matrix modes which mainly include concentrated matrix, AB matrix, star-shaped matrix, dandelion matrix and triangular matrix ${ }^{[3]}$.

Wuliangye can establish binary star microblog matrix which owns two microblog centers, including enterprise official microblog and enterprise senior management microblog. Such matrix is suitable for senior management with strong influence in the society or industry. Microblog influence can expand through enterprise senior management. Wuliangye microblog matrix is microblog cluster centered by "Wuliangye official microblog" and "Wuliangye senior management microblog" and surrounded by brand microblog, dealer microblog, sales microblog, product microblog, 
employee microblog and liquor culture museum microblog. Microblogs coordinate mutually.

(II) Design microblog contents and do release planning well

"Says What" in the model refers to microblog marketing contents which impose great influences on microblog marketing effects. Enterprises must elaborately plan microblog contents.

\section{Well design microblog contents}

\section{(1) High relevance of microblog contents}

Due to time fragmentization of microblog users, microblog contents must be highly related to microblog users in order to arouse users' attention. Microblog relevance includes: 1) relevance to user need; design microblog contents according to user demand; 2) relevance to product knowledge; design microblog contents according to extended knowledge of products; 3) relevance to enterprise objective; microblog contents aim to achieve certain enterprise objective ${ }^{[4]}$.

(2) Cautiously involve microblog contents

Since religion, nationality and political issues have high sensibility, microblog topics should avoid such contents as far as possible.

\section{Do microblog release planning well}

\section{(1) Content sequence planning}

Microblog fragmentization feature means the microblog published by enterprises is fleeting. Due to short microblog contents, a piece of microblog cannot sufficiently explain one thing. So, the legal and theme must be used to classify fragment microblog contents. Thus, please attach importance to label and topic setting to make fragment microblog contents become complete and orderly.

\section{(2) Release time planning}

Based on the research of business enterprise research group of Sina Microblog, the quantity of microblog forwarding and commenting is different for the microblog released at different points-in-time. Users are more active in Friday, Saturday and Sunday. The number of online users will present the peak during 12:00-13:00, 16:00-17:00 and 22:00-23:00. Thus, it is better to release enterprise microblog at these points-in-time.

\section{Design of Wuliangye microblog contents}

Previously, the microblog of many liquor enterprises aimed to publicize enterprise culture and release enterprise news and promotion information. Currently, it is a diversified era. Wuliangye microblog marketing should be conducted toward diversification. Microblog can be used to publicize enterprise culture, explain brand orientation, packaging and culture of Wuliangye, Wuliangchun and Wuliangchun, popularize long-standing liquor culture, comment social events, and introduce investment projects of enterprises.

\section{(III) Select microblog platform and utilize celebrity effect}

"In Which Channel" in the model refers to selection of communication media during microblog marketing, including selection of microblog platform and microblog content presenting mode.

\section{Select microblog platform}

Currently, Chinese mainstream microblog platforms include Sina Microblog, Tencent Microblog, Netease Microblog, Sohu Microblog and Baidu Microblog. The market share and active users of Sina Microblog are much higher than that of other microblogs. Wuliangye can choose Sina Microblog as microblog release platform. Other liquor enterprises mostly choose Sina Microblog.

\section{Utilize celebrity effect}

In marketing, celebrity effect is often utilized to achieve marketing campaigns. Wuliangye microblog can utilize external stars or enterprise celebrities, such as enterprise senior manager and star wine mixing masters to improve microblog marketing effects.

(IV) Features of Wuliangye microblog

"To Whom" in the model refers to the audiences of microblog marketing. During microblog marketing, it is necessary to design microblog features according to audiences.

(1) User orientation

Wuliangye microblog orientation should keep consistent with Wuliangye brand orientation.

(2) Microblog features

It can be seen from the above analysis that microblog users are mainly highly-educated young 
people. To adapt their needs, Wuliangye microblog should be fashionable, concise, interesting, original and personalized.

(V) Enhance microblog interaction and improve microblog effects

"With What Effect" in the model refers to effect analysis of microblog marketing. Enterprises should evaluate their microblog and influence in real time and continuously perfect enterprise microblog.

\section{Evaluation indexes of microblog marketing effect}

Enterprise microblog often focuses on the number of followers, fans, forwarding and collection rather than the number of microblogs to evaluate microblog value and influence. Enterprises need to pay attention to these indexes anytime. The data center of Sina Microblog can refer to these indexes very conveniently.

\section{Enhance interactions and boost effects}

Wuliangye needs to set up a special department and arrange specially-assigned persons to tale charge of microblog information release and comment reply and regularly collect the changes of fans' attention so as to adjust microblog contents, enhance interactions with fans and improve microblog marketing effects.

\section{References}

[1] Qiao Jinxing, Enterprise microblog: new brand marketing tool [J]. Today's Massmedia (Academic Edition), 2010 (5): 14016

[2] Chu Yaling,Core of "sublation" of new media communication theory [J]. Journal of Tangshan College, 2012 (5): 40-43

[3] Chen Ran, Liu Qiong, Problems and strategies of academic periodical microblog marketing case study of academic periodical of journalism and communication [J]. Youth Journalist, 2013 (17): 69-70

[4] Yan Xing, Chang Yaping, Overview of microblog research [J]. Journal of Information, 2011(9): 61-66 